\title{
INTERMITTENCY IN THE TRANSITION TO TURBULENCE*
}

\author{
A. C. FOWLER ${ }^{\dagger}$ AND P. D. HOWELL ${ }^{\dagger}$
}

\begin{abstract}
It is commonly known that the intermittent transition from laminar to turbulent flow in pipes occurs because, at intermediate values of a prescribed pressure drop, a purely laminar flow offers too little resistance, but a fully turbulent one offers too much. We propose a phenomenological model of the flow, which is able to explain this in a quantitative way through a hysteretic transition between laminar and turbulent "states," characterized by a disturbance amplitude variable that satisfies a natural type of evolution equation. The form of this equation is motivated by physical observations and derived by an averaging procedure, and we show that it naturally predicts disturbances having the characteristics of slugs and puffs. The model predicts oscillations similar to those which occur in intermittency in pipe flow, but it also predicts that stationary "biphasic" states can occur in sufficiently short pipes.
\end{abstract}

Key words. intermittency, transition, turbulence, slugs, puffs

AMS subject classifications. $76 \mathrm{E} 30,76 \mathrm{~F} 10$

PII. S0036139900368893

1. Introduction. Ever since Reynolds's (1883) seminal paper on the transition to turbulence in pipe flow, it has been known that the transition occurs in an intermittent fashion. As the Reynolds number increases beyond a value of around 2000 (although the precise value depends on the pipe used and on the experimental conditions at the inlet), intermittent flashes of turbulence can be seen in the pipe. Furthermore, the reason for this intermittency is well known, at least in a crude way (Prandtl and Tietjens (1934, pp. 36f.)). Turbulent flow at a given flow rate has a higher drag than laminar flow, and so, as the pressure drop driving the flow is increased, there arises a critical interval of flow rate within which laminar flow offers too low a resistance to the pressure drop but turbulent flow provides too high a resistance. In this intermediate case, the flow cycles between the two types of flow, and this is manifested in the pipe through the regular occurrence of turbulent "flashes"; this is Reynolds's term, but it has now become more customary to call the flashes "slugs" or "puffs" (Wygnanski and Champagne (1973)), depending on their provenance. The resultant flow then oscillates, producing an oscillatory (and indeed, periodic) outlet flow (Prandtl and Tietjens (1934, p. 37)).

It is perhaps unsurprising that there have been few attempts to recover these observations theoretically. Of necessity, any putative model must be semiempirical, and those that have been put forward (Bohr and Rand (1991), Deissler (1987a,b), Sakaguchi and Brand (1996)) serve as qualitative analogues rather than quantitative ones, and their aim has been to explain qualitatively the existence of turbulent slugs, rather than to draw a quantitative comparison; in addition, the resulting periodic solutions have not been found, although Deissler (1987b) hints at a mechanism similar to that suggested here.

Our aim in this paper is to provide a simple model which avoids the detailed complexities of three-dimensional turbulent flow, but which is nevertheless built solidly on

\footnotetext{
* Received by the editors March 10, 2000; accepted for publication (in revised form) August 9, 2002; published electronically March 26, 2003.

http://www.siam.org/journals/siap/63-4/36889.html

†Mathematical Institute, Oxford University, 24-29 St Giles', Oxford OX1 3LB, England (fowler@maths.ox.ac.uk, howell@maths.ox.ac.uk).
} 
observed features of turbulence, and in particular, on the experimentally determined drag law. Although our motivating aim is to provide a predictive mechanism for intermittency and flow oscillations, we also find that we can make detailed comparisons with slug and puff dynamics. Wygnanski and Champagne (1973) distinguished these on the basis that slugs occurred at higher Reynolds number (above about 2700), were caused by low amplitude disturbances, and spread longitudinally as they propagated, the front and rear travelling at speeds respectively greater and less than the mean flow speed $u$, at least for large enough Reynolds number. In a very careful study, Lindgren (1957) showed that the front and rear wave speeds of slugs appeared to approach a value of about $0.9 u$ as the Reynolds number decreased towards a value $R_{k}$ (equal to about 2400 in his experiments), although in fact distinct slug measurements could be made only down to Reynolds number 2700.

At lower Reynolds number (below 2700 in Wygnanski and Champagne's experiments, presumably below 2400 in Lindgren's), puffs are seen. In contrast to slugs, puffs are generated by large amplitude disturbances at the inlet, and unlike slugs, which have relatively sharp leading and trailing edges, puffs have only a sharp trailing edge and a diffuse front. The trailing edge migrates backwards relative to the mean flow, with the difference between their two speeds tending to zero as the Reynolds number decreases towards a value $\underline{R}$ (about 2050 in Lindgren's experiments). Lindgren identifies a further Reynolds number $\bar{R}_{k}$, above which slugs grow as they propagate; presumably this is the Reynolds number at which stable slugs become viable. A final critical value is $\bar{R}$, above which fully developed turbulence can be maintained throughout the pipe.

\section{A model for intermittency in turbulent flow.}

2.1. Behavior of the wall friction. The Reynolds number for flow in a pipe of diameter $d$ is

$$
R e=\frac{\rho u d}{\mu},
$$

where $u$ is the mean flow velocity, $\rho$ is the density, and $\mu$ is the viscosity. In a pipe of length $l$, the pressure drop along its length in conditions of steady flow is given by

$$
F=\frac{\Delta p}{l}=\frac{\lambda \rho u^{2}}{2 d},
$$

where the drag coefficient $\lambda$ is a function of the Reynolds number (Schlichting (1979)). In conditions of fully developed laminar flow, which pertain for $R e \lesssim 2300$,

$$
\lambda_{L}=\frac{64}{R e},
$$

whereas Blasius's (1913) empirical relation for fully developed turbulent flow is

$$
\lambda_{T}=\frac{0.3164}{R e^{1 / 4}}
$$

and is approximately valid for $3300<R e<10^{5}$. At higher Reynolds number, a more sophisticated result based on Prandtl's mixing length theory can be used to define $\lambda$ implicitly, but (2.4) will suffice for the present purpose, where such high Reynolds numbers are not relevant. 


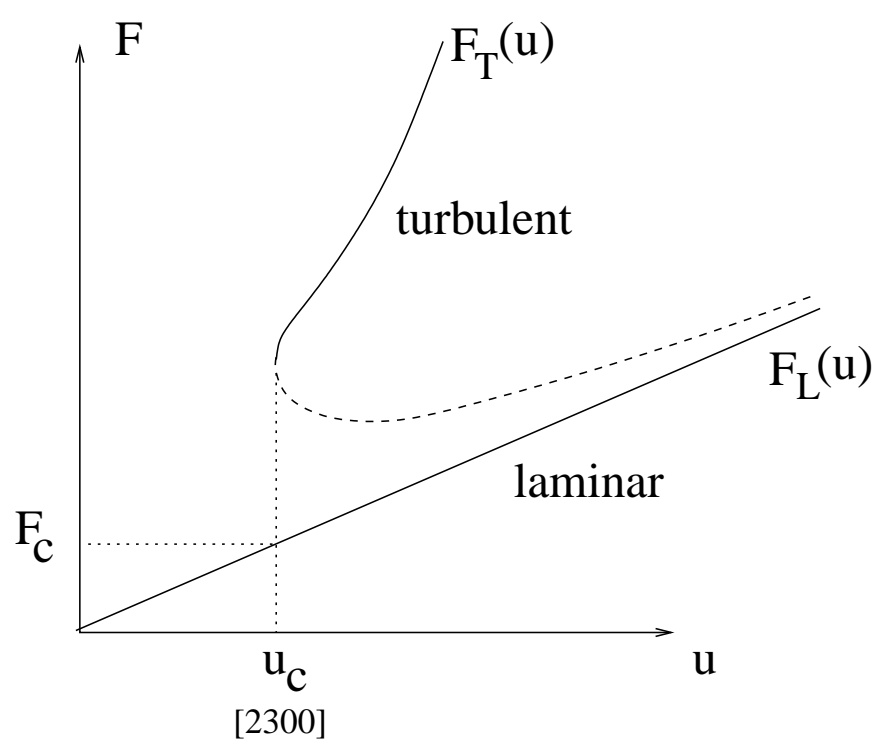

Fig. 1. Schematic drag law for laminar and turbulent flow. The experimentally determined laminar and turbulent values are the solid curves, and we hypothesize that an unstable (dashed) branch separates the two, as shown.

Between the onset of transition at $R e=2300$ and the attainment of fully developed turbulence (throughout the pipe) at $R e \approx 3300,{ }^{1}$ there is a region in which $\lambda$ increases with Re. This odd behavior is associated with the phenomenon of intermittency. At Reynolds numbers above 2300, fluid can exist locally in a turbulent state, but for $R e<3300$, turbulent slugs of fluid are interspersed with laminar plugs. The intermittency factor $\gamma$ (the fraction of time at a fixed location for which the flow is turbulent) grows with distance downstream, and also with Reynolds number (Rotta (1956), Wygnanski and Champagne (1973)).

We wish to place a specific interpretation on this observed behavior. The local cross-sectionally averaged wall friction in a tube of diameter $d$ is given from (2.2) by

$$
F_{L}=\frac{32 \mu u}{d^{2}}
$$

for laminar flow (using (2.3)) and

$$
F_{T} \approx \frac{0.16 \rho^{3 / 4} \mu^{1 / 4} u^{7 / 4}}{d^{5 / 4}}
$$

for turbulent flow.

As shown in Figure 1, the turbulent friction $F_{T}(u)$ exists as a local description down to Reynolds numbers of 2300 , while the laminar expression $F_{L}(u)$ exists as a solution for all values of $u$. In particular, at values of $R e$ above 2300 , both behaviors are possible as locally stable solutions of the Navier-Stokes equation.

\footnotetext{
${ }^{1}$ This numerical value and that of the "onset" at 2300 depend on the level of the inlet disturbance, as well as the particular experimental set-up; these values are adopted from inspection of experimental drag measurements - see Schlichting (1979, Figure 20.1) - and will be used as typical values. In due course, we will relate them to the critical values discussed by Lindgren (1957).
} 
Although it is not in fact essential to our argument, the existence of a jump in $F$ between the two accessible solution branches suggests strongly that there is a third intermediate branch which joins the laminar and turbulent branches, as shown in Figure 1, and this is supported by other experimental work also (Huang and Huang (1989), Wygnanski and Champagne (1973), Darbyshire and Mullin (1995)). Moreover, the existence of an unstable intermediate branch is analogous to the existence of unstable equilibria in the transition to turbulence of plane and pipe Poiseuille flow (Orszag and Patera (1980), (1983)), and this lends support to the concept embodied in Figure 1.

We take the form of the dashed part of the $F(u)$ curve in Figure 1 as a hypothesis. It is then convenient to think of the local friction $F$ as a state variable (rather like enthalpy), and to suppose that it is a measure of the laminar or turbulent "phase" of the fluid. And, rather like a phase change, the intermediate state is not accessible. In a fluid, boiling leads to dispersed phases at intermediate enthalpies, and transition to turbulence leads to intermittency at intermediate flow rates.

From the point of view of dynamical systems theory, the existence of a state variable $F$ demarcating laminar and turbulent phases with a multiple valued equilibrium suggests that the simplest model beyond the mixing length theory which can describe transitions between laminar and turbulent states is one which embodies an evolution equation for $F$. In order to see how such an equation might be proposed, we need to study the way in which the basic mixing length theory produces the equilibrium structure of Figure 1.

2.2. Averaging. We start with the Navier-Stokes equations

$$
\begin{aligned}
\frac{\partial u_{i}}{\partial x_{i}} & =0 \\
\rho\left[\frac{\partial u_{i}}{\partial t}+\frac{\partial}{\partial x_{j}}\left(u_{i} u_{j}\right)\right] & =-\frac{\partial p}{\partial x_{i}}+\mu \nabla^{2} u_{i} .
\end{aligned}
$$

Following common procedure (Mathieu and Scott (2000)), we define

$$
u_{i}=\bar{u}_{i}+u_{i}^{\prime}
$$

where $\bar{u}_{i}$ is a local time average of $u_{i}$, and $u_{i}^{\prime}$ represents the fluctuating part. More specifically,

$$
\bar{u}_{i}=\frac{1}{2 T} \int_{t-T}^{t+T} u_{i} d t
$$

and we suppose formally that $u_{i}^{\prime}$ varies on a time scale $\ll T$, while we allow $\bar{u}_{i}$ to vary on times $\gg T$. (The necessity for this assumption can be removed by taking ensemble averages instead.)

Averaging of (2.7) thus leads to

$$
\begin{aligned}
\frac{\partial \bar{u}_{i}}{\partial x_{i}} & =0 \\
\rho\left[\frac{\partial \bar{u}_{i}}{\partial t}+\frac{\partial}{\partial x_{j}}\left(\bar{u}_{i} \bar{u}_{j}\right)\right] & =-\frac{\partial \bar{p}}{\partial x_{i}}+\mu \nabla^{2} \bar{u}_{i}+\frac{\partial}{\partial x_{j}}\left(-\rho \overline{u_{i}^{\prime} u_{j}^{\prime}}\right) .
\end{aligned}
$$

Next we define the cross-sectional average over the pipe as

$$
\hat{h}=\frac{1}{S} \int_{S} h d S,
$$


where $S$ denotes the cross-sectional area. If we let $x\left(=x_{1}\right)$ denote distance down the pipe axis, then also

$$
\frac{\widehat{\partial f_{j}}}{\partial x_{j}}=\frac{\partial \hat{f}_{1}}{\partial x}-\frac{1}{R}\left\langle f_{n}\right\rangle_{w}
$$

where $R=S / P$ is the hydraulic radius, $P$ is the pipe perimeter, $f_{n}=\mathbf{f} . \mathbf{n}$ denotes the inwards normal component of $\mathbf{f}$ ( $\mathbf{n}$ is the unit inward normal) and \langle\rangle$_{w}$ denotes the circumferential average,

$$
\langle g\rangle_{w}=\frac{1}{P} \int_{\partial S} g d s .
$$

We take a cross-sectional average of (2.10). We must have $\left\langle\bar{u}_{n}\right\rangle_{w}=0$, thence $\partial \hat{\bar{u}}_{1} / \partial x=0$; i.e., the mean flow is

$$
\hat{\bar{u}}_{1}=u(t)
$$

say, and thus, considering the $x$ component only,

$$
\rho \dot{u}=-\frac{\partial p}{\partial x}-F+\frac{\partial}{\partial x}\left(-\widehat{\widehat{u_{1}^{\prime 2}}}\right),
$$

where we write $\hat{\bar{p}}=p$, and

$$
F=\frac{\mu}{R}\left\langle\frac{\partial \bar{u}_{1}}{\partial n}\right\rangle_{w}+\frac{1}{R}\left\langle-\rho \overline{u_{1}^{\prime} u_{n}^{\prime}}\right\rangle_{w}
$$

Strictly, the second term on the right-hand side of (2.16) can be neglected, since $u_{i}^{\prime}=0$ at the wall. However, it is more common to define the "wall" in (2.13) to lie just outside the laminar sublayer, so that although $\overline{\mathbf{u}} \approx 0$ there, we allow the Reynolds stresses $\left(-\rho \overline{u_{1}^{\prime} u_{j}^{\prime}}\right)$ to be nonzero at the wall. (In particular, this allows us to deal with rough walls.) We follow this practice here.

In conditions of steady uniform flow, (2.16) defines the wall drag, and Figure 1 represents the observed variation of $F$ with $u$. In laminar flow,

$$
\left\langle\frac{\partial \bar{u}_{1}}{\partial n}\right\rangle_{w}=\frac{8 u}{d}
$$

while Prandtl's mixing length theory for turbulent flow also leads to $\left\langle\partial \bar{u}_{1} / \partial n\right\rangle_{w} \propto u / d$, although with a different coefficient. We will suppose that (2.17) applies in both cases, partly for simplicity, and partly because the laminar contribution is small in turbulent flow, so that the inaccuracy of (2.17) in that case is inconsequential. (A more realistic prescription for $\left\langle\partial \bar{u}_{1} / \partial n\right\rangle_{w}$ would be $L(A) u / d$, but we will persevere with (2.17).)

Given our constitutive prescription (2.17) for $\left\langle\partial \bar{u}_{1} / \partial n\right\rangle_{w}$, we now define a fluctuation velocity

$$
A=\frac{d}{8 \mu}\left\langle-\rho \overline{u_{1}^{\prime} u_{n}^{\prime}}\right\rangle_{w} .
$$

It follows from (2.16) and (2.17) that (since the hydraulic radius of a pipe is $d / 4$ )

$$
F=\frac{8 \mu}{d R}[u+A]
$$




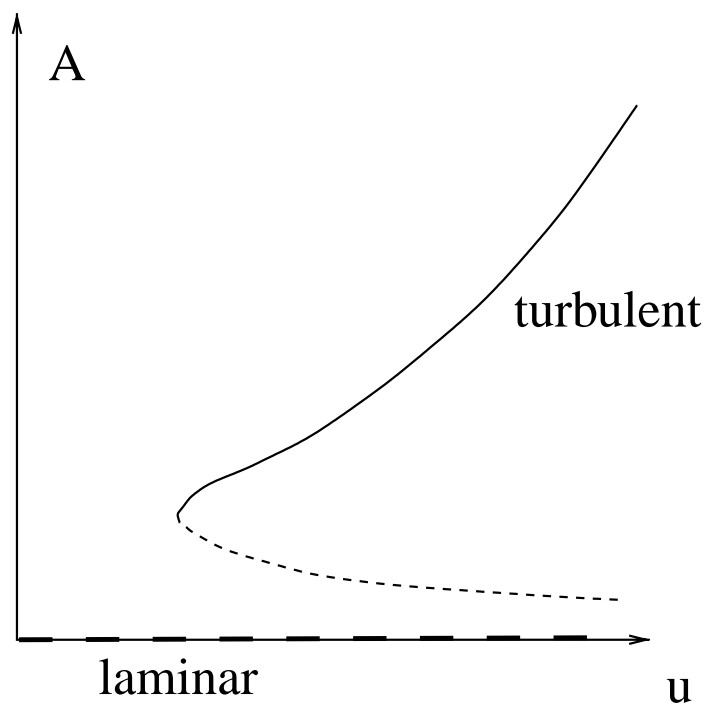

FIG. 2. Diagram equivalent to Figure 1 for the postulated equilibrium, showing "amplitude" A versus flow rate u (or equivalently, Reynolds number).

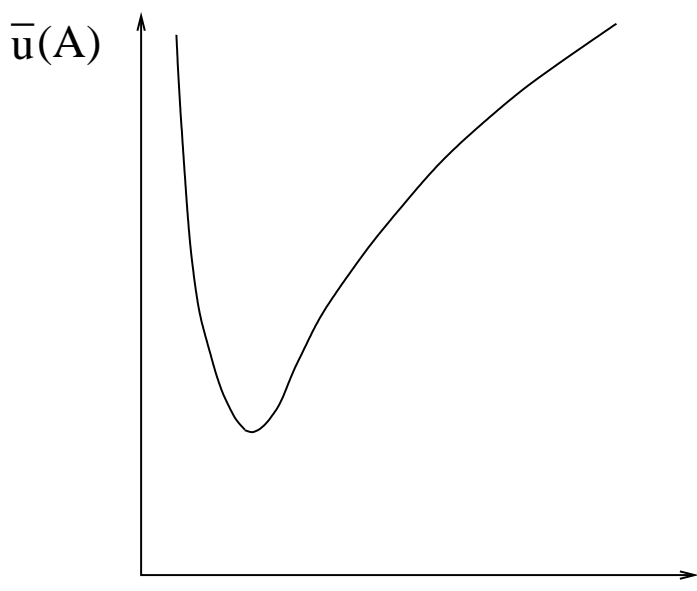

A

FIG. 3. The function $\bar{u}(A)$ defined by reversing the axes in Figure 2.

In terms of $A$, the equilibrium friction diagram Figure 1 now takes the form shown in Figure 2, and thus defines a single valued function $\bar{u}(A)$, such that in Figure 2, $u=\bar{u}(A)$ for $A \neq 0$, as shown in Figure 3 .

The closure problem of turbulence is that of determining the Reynolds stresses $\left(-\rho \overline{u_{i}^{\prime} u_{j}^{\prime}}\right)$. The idea of an eddy viscosity $\mu_{T}$ involves the closure assumption

$$
-\rho \overline{u_{i}^{\prime} u_{j}^{\prime}}=\mu_{T}\left(\frac{\partial \bar{u}_{i}}{\partial x_{j}}+\frac{\partial \bar{u}_{j}}{\partial x_{i}}\right)-\frac{2}{3} \rho k \delta_{i j}
$$


where

$$
k=\frac{1}{2} \overline{u_{i}^{\prime} u_{i}^{\prime}}
$$

is the turbulent kinetic energy per unit mass. In unidirectional flows, Prandtl's mixing length hypothesis takes the form

$$
\mu_{T} \propto \rho n^{2}\left|\frac{\partial \bar{u}_{1}}{\partial n}\right|,
$$

$n$ representing distance from the wall. In a circular pipe, Schlichting (1979) shows that this empirical assumption actually leads to virtually perfect agreement with experimental measurements of wall drag. That is to say, in a steady uniform flow, $F$ given by (2.16) together with an empirical assumption of eddy viscosity type for turbulent flow (and the equivalent exact molecular viscosity rule for laminar flow) is able to match the upper and lower branches in Figure 1 very well.

Prescription of (2.20) with (2.22) is an example of a zero-dimensional model closure. Such a closure is not good for three-dimensional flows, and this led to the development of more complicated "one-equation" or "two-equation" models. A typical example is the famous " $k-\varepsilon$ " model (Mathieu and Scott (2000)), in which (2.20) is still used; one assumes (for example) that

$$
\mu_{T}=\frac{C k^{2}}{\varepsilon_{D}},
$$

where

$$
\varepsilon_{D}=\frac{\mu}{2 \rho} \overline{\left(\frac{\partial u_{i}^{\prime}}{\partial x_{j}}+\frac{\partial u_{j}^{\prime}}{\partial x_{i}}\right)\left(\frac{\partial u_{i}^{\prime}}{\partial x_{j}}+\frac{\partial u_{j}^{\prime}}{\partial x_{i}}\right)}
$$

is the rate of dissipation of turbulent kinetic energy, and the model is closed by posing two evolution equations for $k$ and $\varepsilon_{D}$ (hence the term, two-equation model). In view of its similarity to the model we propose below, we give an example of a typical closure for $k$ :

$$
\frac{\partial k}{\partial t}+\bar{u}_{k} \frac{\partial k}{\partial x_{k}}=\Pi-\varepsilon_{D}+\frac{\partial}{\partial x_{k}}\left(\frac{\mu_{T}}{\sigma \rho} \frac{\partial k}{\partial x_{k}}\right) ;
$$

$\Pi$ is prescribed in terms of $\bar{u}, \sigma$ is a constant. Note the basic type of advection-diffusion equation with source and sink terms.

In an analogous way, our purpose now is to consider how one might model slow time and space evolution of the fluctuation amplitude $A$. Derivation of an equation for $A$ is not easy, nor is it our main purpose, and we will confine ourselves to providing a motivation for the form such an equation might take. We begin with the equations for the fluctuations $u_{i}^{\prime}$,

$$
\begin{gathered}
\frac{\partial u_{i}^{\prime}}{\partial x_{i}}=0 \\
\rho\left[\frac{\partial u_{i}^{\prime}}{\partial t}+\frac{\partial}{\partial x_{k}}\left\{\bar{u}_{i} u_{k}^{\prime}+u_{i}^{\prime} \bar{u}_{k}+u_{i}^{\prime} u_{k}^{\prime}-\overline{u_{i}^{\prime} u_{k}^{\prime}}\right\}\right]=-\frac{\partial p^{\prime}}{\partial x_{i}}+\mu \nabla^{2} u_{i}^{\prime} .
\end{gathered}
$$


Multiplying the second equation by $u_{j}^{\prime}$ and its $u_{j}^{\prime}$ equivalent by $u_{i}^{\prime}$, adding the two, and time averaging leads to the evolution equation for the Reynolds stress tensor

$$
R_{i j}=-\rho \overline{u_{i}^{\prime} u_{j}^{\prime}}
$$

in the form (see Launder, Reece, and Rodi (1975))

$$
\begin{aligned}
\frac{\partial R_{i j}}{\partial t}+\frac{\partial}{\partial x_{k}}\left[\bar{u}_{k} R_{i j}\right]= & -\left[R_{j k} \frac{\partial \bar{u}_{i}}{\partial x_{k}}+R_{i k} \frac{\partial \bar{u}_{j}}{\partial x_{k}}\right]+2 \mu \overline{\nabla u_{i}^{\prime} \cdot \nabla u_{j}^{\prime}}-\overline{p^{\prime}\left(\frac{\partial u_{i}^{\prime}}{\partial x_{j}}+\frac{\partial u_{j}^{\prime}}{\partial x_{i}}\right)} \\
& +\frac{\partial}{\partial x_{k}}\left(\rho \overline{u_{i}^{\prime} u_{j}^{\prime} u_{k}^{\prime}}\right)+\frac{\partial}{\partial x_{k}}\left[\overline{p^{\prime}\left(u_{i}^{\prime} \delta_{j k}+u_{j}^{\prime} \delta_{i k}\right)}\right]+\frac{\mu}{\rho} \nabla^{2} R_{i j} .
\end{aligned}
$$

Launder, Reece, and Rodi (1975) characterize the terms in this equation in the following way. Of the six terms on the right-hand side of (2.28), the first represents generation and the second, dissipation; the last three represent transport, while the fourth (pressure strain) term is characterized by a spatial integral of the fluctuating velocity field. To see this, we take the divergence of the Navier-Stokes equation to find

$$
\nabla^{2} p=-\rho \frac{\partial^{2}\left(u_{i} u_{j}\right)}{\partial x_{i} \partial x_{j}}
$$

whence the fluctuating pressure field $p^{\prime}$ satisfies

$$
\nabla^{2} p^{\prime}=-\rho \frac{\partial^{2}}{\partial x_{i} \partial x_{j}}\left[\bar{u}_{i} u_{j}^{\prime}+u_{i}^{\prime} \bar{u}_{j}+u_{i}^{\prime} u_{j}^{\prime}-\overline{u_{i}^{\prime} u_{j}^{\prime}}\right]
$$

and $p^{\prime}$ can be written as a spatial integral convolving a suitable Green's function with the right-hand side of $(2.30)$.

From (2.18), $A=(d / 8 \mu)\left\langle R_{1 n}\right\rangle_{w}$, and in the absence of swirling motion, $\langle g\rangle_{w}=g$ for any of the terms $g$ in (2.28). Also, on the wall $\bar{u}_{i}=0$, and $\partial \bar{u}_{1} / \partial n$ is the only nonvanishing velocity derivative term $\left(\partial \bar{u}_{n} / \partial n=-\partial \bar{u}_{1} / \partial x=0\right)$. Setting $i=1$, $j=n$, a wall average leads to

$$
\begin{aligned}
\frac{\partial}{\partial t}\left\langle-\rho \overline{u_{1}^{\prime} u_{n}^{\prime}}\right\rangle_{w}+ & \frac{\partial}{\partial x}\left\langle-\rho \overline{u_{1}^{\prime 2} u_{n}^{\prime}}\right\rangle_{w} \\
= & \left\langle\rho \overline{u_{n}^{\prime 2}}\right\rangle_{w}\left\langle\frac{\partial \bar{u}_{1}}{\partial n}\right\rangle_{w}+2 \mu\left\langle\overline{\nabla u_{1}^{\prime} \cdot \nabla u_{n}^{\prime}}\right\rangle_{w} \\
& -\left\langle\frac{\partial}{\partial n}\left(-\rho \overline{u_{1}^{\prime} u_{n}^{\prime 2}}\right)-\overline{u_{1}^{\prime} \frac{\partial p^{\prime}}{\partial n}}+\overline{p^{\prime} \frac{\partial u_{1}^{\prime}}{\partial n}}+\frac{\mu}{\rho} \frac{\partial^{2}}{\partial n^{2}}\left(-\rho \overline{u_{1}^{\prime} u_{n}^{\prime}}\right)\right\rangle_{w} \\
& +\frac{\partial}{\partial x}\left\langle\overline{p^{\prime} u_{n}^{\prime}}\right\rangle_{w}+\frac{\mu}{\rho} \frac{\partial^{2}}{\partial x^{2}}\left\langle-\rho \overline{u_{1}^{\prime} u_{n}^{\prime}}\right\rangle_{w} .
\end{aligned}
$$

It is not so easy to characterize the nature of the terms in (2.31) as we did for (2.28), because some of the transport terms in (2.28) involving normal derivatives can no longer be so categorized. Such terms, together with the pressure strain terms, form the third term on the right-hand side of (2.31). The other terms have direct analogy with those in (2.28). The second term on the left-hand side of (2.31) is a transport term and arises from the fourth term on the right-hand side of (2.28). The first and second terms on the right-hand side of (2.31) are source and sink terms, arising from the corresponding generation and dissipation terms in (2.28). The final two terms in (2.31) are transport terms. 
2.3. Closure assumptions. We now face the daunting task of choosing constitutive laws for the various terms in (2.31). In choosing an eddy viscosity model, we specifically supposed (cf. (2.20))

$$
\left\langle-\rho \overline{u_{1}^{\prime} u_{n}^{\prime}}\right\rangle_{w}=\left.\mu_{T}\left(\frac{\partial \bar{u}_{1}}{\partial n}+\frac{\partial \bar{u}_{n}}{\partial x}\right)\right|_{w}
$$

where $\mu_{T}$ itself depends on $\bar{u}_{1}$. In uniform flow $(\partial / \partial t=\partial / \partial x=0)$, this subsequently leads via Prandtl's mixing length theory to a functional relation between $u$ and $A$. Given that we suppose $u$ and $A$ to be defined via (2.17) and (2.18), that is,

$$
u=\frac{d}{8}\left\langle\frac{\partial \bar{u}_{1}}{\partial n}\right\rangle_{w}, \quad A=\frac{d}{8 \mu}\left\langle-\rho \overline{u_{1}^{\prime} u_{n}^{\prime}}\right\rangle_{w}
$$

it is natural to relate fluctuating terms in (2.31) to $A$, and terms in $\bar{u}_{1}$ to $u$. Furthermore, if we neglect $\partial / \partial t$ and $\partial / \partial x$, we should regain the equilibrium curve (with $A>0$ ) of Figure 2, which we will suppose is written in the form $u=\bar{u}(A)$. At this point, we specifically ignore the problem of constituting the pressure fluctuation terms. As discussed above, we expect such terms to give rise to convolution integrals, but their omission will clarify the subsequent discussion without compromising the results. The above comments suggest (given (2.33)) that we choose

$$
\begin{aligned}
\left\langle\rho \overline{u_{n}^{\prime 2}}\right\rangle_{w}\left\langle\frac{\partial \bar{u}_{1}}{\partial n}\right\rangle_{w} & -\left\langle\frac{\partial}{\partial n}\left(-\rho \overline{u_{1}^{\prime} u_{n}^{\prime 2}}\right)-2 \mu \bar{\nabla} \overline{u_{1}^{\prime} \cdot \nabla u_{n}^{\prime}}+\frac{\mu}{\rho} \frac{\partial^{2}}{\partial n^{2}}\left(-\rho \overline{u_{1}^{\prime} u_{n}^{\prime}}\right)\right\rangle_{w} \\
& =\frac{8 \mu}{d} r(A)[u-\bar{u}(A)],
\end{aligned}
$$

where

$$
r(A)=\frac{1}{\mu}\left\langle\rho \overline{u_{n}^{\prime 2}}\right\rangle_{w}
$$

is a positive function, with $d r / d A>0$ and $r=0$ when $A=0$. The last term in (2.31) is a laminar diffusion term,

$$
\frac{\mu}{\rho} \frac{\partial^{2}}{\partial x^{2}}\left\langle-\rho \overline{u_{1}^{\prime} u_{n}^{\prime}}\right\rangle_{w}=\frac{\mu}{\rho} \cdot \frac{8 \mu}{d} \frac{\partial^{2} A}{\partial x^{2}},
$$

and it is plausible to expect an equivalent turbulent diffusive term to exist also.

There are two ingredients that we might expect to find in $\left\langle-\rho \overline{u_{1}^{\prime 2} u_{n}^{\prime}}\right\rangle_{w}$. If we follow the (apparently arbitrary) recipe (2.32), which relates $\left\langle\overline{u_{1}^{\prime} u_{n}^{\prime}}\right\rangle_{w}$ to $\partial \bar{u}_{1} / \partial n$ and $\partial \bar{u}_{n} / \partial x$, we would equivalently write

$$
\left\langle\overline{\left(-\rho u_{1}^{\prime} u_{n}^{\prime}\right) u_{1}^{\prime}}\right\rangle_{w}=-\frac{\mu_{T}}{\rho} \frac{\partial}{\partial x}\left\langle-\rho \overline{u_{1}^{\prime} u_{n}^{\prime}}\right\rangle_{w}+\cdots
$$

but also

$$
\left\langle-\rho \overline{u_{1}^{\prime 2} u_{n}^{\prime}}\right\rangle_{w}=\mu_{T} \frac{\partial}{\partial n}\left\langle\overline{u_{1}^{\prime 2}}\right\rangle_{w}+\cdots
$$

The simplest choice is then

$$
\left\langle-\rho \overline{u_{1}^{\prime 2} u_{n}^{\prime}}\right\rangle_{w}=\frac{\mu_{T}}{\rho} \frac{\partial}{\partial x}\left\langle-\rho \overline{u_{1}^{\prime} u_{n}^{\prime}}\right\rangle_{w}+\mu_{T} \frac{\partial}{\partial n}\left\langle\overline{u_{1}^{\prime 2}}\right\rangle_{w} .
$$


Finally, it seems reasonable to propose

$$
\mu_{T} \frac{\partial}{\partial n}\left\langle\overline{u_{1}^{\prime 2}}\right\rangle_{w}=\frac{8 \mu}{d} W(A, u)
$$

with $W \geq 0$ as we expect $\partial \overline{u^{\prime 2}} / \partial n$ to be positive at the wall: as for $r(A)$, we expect $W=0$ when $A=0$ and $\partial W / \partial A>0$.

Multiplying (2.31) by $d / 8 \mu$, we have (ignoring the pressure fluctuation terms)

$$
\begin{aligned}
\frac{\partial A}{\partial t} & +\frac{\partial}{\partial x}\left[-\frac{\mu_{T}}{\rho} \frac{\partial A}{\partial x}+W(A, u)\right] \\
& =r(A)[u-\bar{u}(A)]+\frac{\mu}{\rho} \frac{\partial^{2} A}{\partial x^{2}} .
\end{aligned}
$$

The functions $W(A, u)$ and $r(A)$ in (2.41) are matters of conjecture. To be specific, we now define

$$
U=\frac{\partial W}{\partial A}=\frac{\partial\left[\mu_{T} \frac{\partial}{\partial n}\left\langle\overline{u_{1}^{\prime 2}}\right\rangle_{w}\right]}{\partial\left\langle-\rho \overline{u_{1}^{\prime} u_{n}^{\prime}}\right\rangle_{w}},
$$

so that $U>0$ is an advection velocity. If we define

$$
D=\frac{\left(\mu+\mu_{T}\right)}{\rho}
$$

in (2.41), then our simple model for $A$ is

$$
A_{t}+U A_{x}=r[u-\bar{u}(A)]+\left(D A_{x}\right)_{x}
$$

where $\bar{u}(A)$ indicates the single valued equilibrium curve with $A \neq 0$ in Figure 2 . This equation represents the idea that $A$ evolves with time while the fluid is advected, seeking the equilibria in Figure 2. The principal difference that a more detailed prescription might make would be the inclusion of integral terms in (2.44) corresponding to the pressure fluctuation terms which we have ignored.

We now discuss suitable choices for $U, r$, and $D$. It is generally thought that pipe Poiseuille flow is linearly stable at all Reynolds numbers (Drazin and Reid (1981)), although the smallest disturbance decay rates tend to zero as $R e \rightarrow \infty$. This will be the case here if $r \bar{u} \propto A$ and $r=o(A)$ as $A \rightarrow 0$. Experimental measurements (Wygnanski and Champagne (1973), Darbyshire and Mullin (1995)) are suggestive of an asymptotic relationship $\bar{u} \sim A^{-2}$ as $\bar{u} \rightarrow \infty$ (see Figure 4), and we will make this assumption here. It then follows that an appropriate choice for $r$ is

$$
r=k A^{3} .
$$

Then (2.44) allows $A=0$ to be linearly stable. An issue here concerns the measurement of experimental disturbance amplitudes. Darbyshire and Mullin (1995) indicate absolute amplitudes, whereas Wygnanski and Champagne's (1973) data (in Figure 4) apparently measures amplitude ratios. There are two points of observation to make. The first is that the data points at higher $R e$ in Figure 4 will place the entire pipe flow in the inlet region, which jeopardizes the interpretation of the amplitude as a perturbation to fully developed flow. The other is that if the amplitude ratio did indeed decrease as weakly as suggested by Figure 4, then the absolute amplitude would 


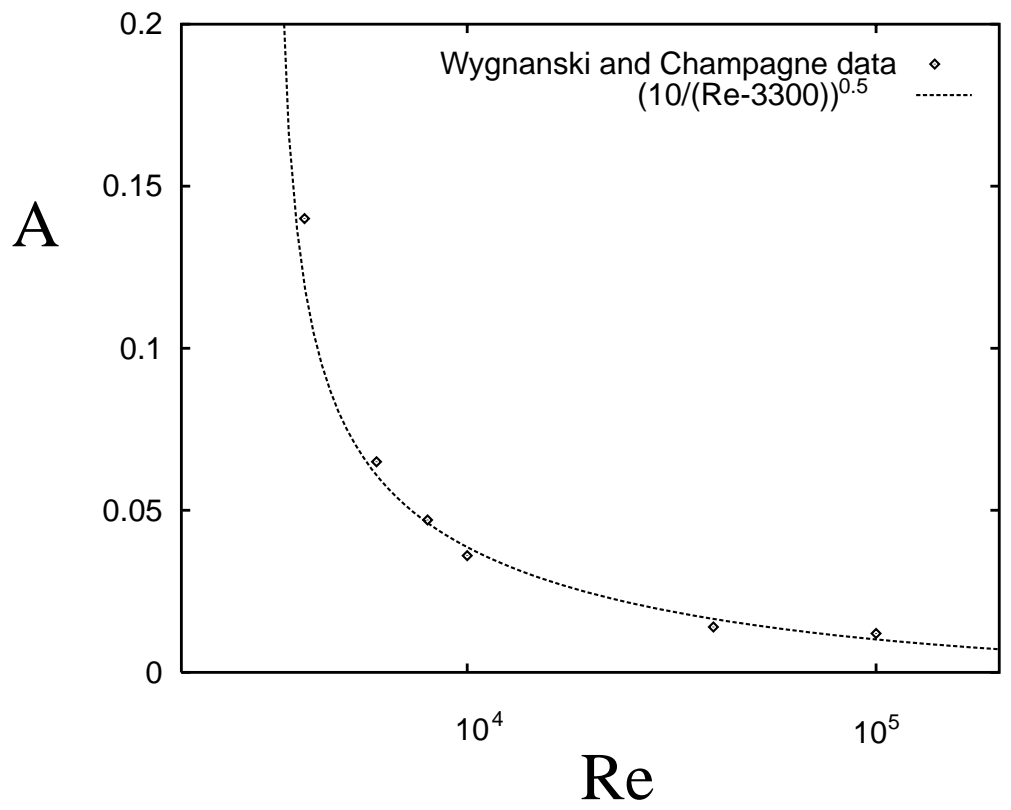

FIG. 4. Data taken by hand from the lower threshold of the slug transition curve of Wygnanski and Champagne (1973) indicating threshold values of $A$ (percent), together with the fitted curve $A=\{10 /(\operatorname{Re}-3300)\}^{1 / 2}$.

eventually increase with $R e$. This does not seem to be suggested by experiment, and is inconsistent with linear theory. We therefore hold to our interpretation of $A$ in (2.44) as an absolute amplitude.

The constant $k$ should represent the idea that the laminar-turbulent transition is associated with a rapid inviscid three-dimensional instability of slowly decaying twodimensional disturbances (Orszag and Patera (1980)). This suggests that $t \sim d / u \sim$ $1 / k A^{2} u$, and it is thus suitable to choose (for example)

$$
k=\frac{1}{[A]^{2} d},
$$

where $[A]$ is a representative value of $A$ near transition.

The amplitude equation (2.44) is then in fact, when $A \ll u$, exactly that which can be derived using multiple scale methods (Davey and Nguyen (1971)), although the correctness of such equations is in doubt (Itoh (1977), Davey (1978)). However, it does seem likely that some such unstable solution branch bifurcates from infinity, as is suggested by the work of Rosenblat and Davis (1979) and Smith and Bodonyi (1982). We do not claim that (2.44) represents the last word in modelling slowly varying fluctuations, but its form is consistent with what is known about pipe flow. As a model, it serves the same purpose as Burgers's (1948) model, and indeed it bears some resemblance, although Burgers's model was more concerned with cross-stream transport of energy. The model (2.44) is also analogous (and serves a similar purpose) to the Swift-Hohenberg-type amplitude model, which has been used by some authors with a purpose similar to that of the present paper (Sakaguchi and Brand (1996), Deissler (1987a)).

The diffusion coefficient $D$ in (2.43) has two parts representative of a laminar 
and a (larger) turbulent diffusivity, and so we take $D \sim u d$ to represent the latter quantity. However, we should also allow $D$ to depend on $A$, since when $A$ is small, $D$ is represented by molecular viscosity. We will find that the diffusive term allows for the existence of convectively growing slugs (Bohr and Rand (1991), Deissler (1987b)), with front and rear ends which travel respectively faster and slower than the mean flow, as is observed in practice.

The choice of a suitable advection velocity $U$ is less clear. Most simply, we would take $U=u$, the mean flow. However, for marginally stable modes at high $R e$, the relevant linear stability advection velocity (Drazin and Reid (1981)) is less than $u$, and we therefore allow $U$ to be less than $u$ and to depend on $A$.

2.4. A model for intermittency. We can then provide a putative model for intermittent turbulent flow as follows. For an incompressible (thus $u=u(t)$ ) fluid flow subject to a mean pressure gradient $F_{m}$ in a pipe $0<x<l$, we solve

$$
A_{t}+U A_{x}=k A^{3}[u-\bar{u}(A)]+\left(D A_{x}\right)_{x} .
$$

In $(2.15)$ we neglect the term $\frac{\partial}{\partial x}\left(-\widehat{\widehat{u_{1}^{\prime 2}}}\right)$, partly because we expect it to be small and partly because the natural constitution of $\overline{u_{1}^{\prime 2}} \propto u^{2}$ leads to its absence, since $u=u(t)$. Then $u$ and $p$ satisfy

$$
\begin{aligned}
\rho \dot{u} & =-p_{x}-F, \\
\int_{0}^{l}-p_{x} d x & =F_{m} l,
\end{aligned}
$$

which together imply (using the definition of $F$ in (2.19))

$$
\rho \dot{u}=F_{m}-\frac{32 \mu}{d^{2}}\left[u+\frac{1}{l} \int_{0}^{l} A d x\right] .
$$

Deissler (1987b) has suggested that if $F_{m}$ is prescribed, then the pressure drop feedback (i.e., (2.49)) may cause intermittency; we will seek to establish this suggestion here.

The pair of equations (2.47) and (2.49) require two boundary conditions for $A$ (as well as initial conditions for $A$ and $u$ ). A primary observation of transition in pipe flow ever since the experiments of Reynolds (1883) is that the level of inlet disturbance is instrumental in determining the nature of the flow. Therefore we prescribe

$$
A=A_{0} \quad \text { at } x=0,
$$

and we expect that the value of $A_{0}$ will be important in determining the dynamics of the flow.

The outlet condition is required only if the diffusion term is included, and, in order to exclude physically inappropriate boundary layers at the channel outlet, we prescribe the passive condition

$$
A_{x}=0 \quad \text { at } x=l
$$

there. 
2.5. Nondimensionalization. We choose scales $[F],[A],[u],[t],[x]$ as follows: $[F]$ is chosen as the critical value $F_{c}=32 \mu u_{c} / d^{2}$ in Figure 1 , where the laminar flow can become unstable to turbulent bursts, and $[u]=u_{c}$ is the corresponding mean flow. Then we choose $[A]=[u],[t]=\rho[u] /[F]$, and $[x]=[u][t]$; the advection velocity $U$ is scaled with $[u]$. We then find that

$$
[x]=\frac{d}{\varepsilon},
$$

where

$$
\varepsilon=\frac{32}{[R e]}
$$

and the Reynolds number scale is

$$
[R e]=\frac{\rho[u] d}{\mu} ;
$$

since $\varepsilon \ll 1(\varepsilon \approx 0.015$ for $[R e]=2300)$, we see that $[x] \gg d$. The scale $[x]$, in fact, describes the inlet region of the pipe (Goldstein (1938, pp. 299f.)). The dimensionless model becomes (using the same notation for the dimensionless variables)

$$
\begin{aligned}
\varepsilon\left[A_{t}+U A_{x}\right] & =A^{3}[u-\bar{u}(A)]+\varepsilon^{2}\left(\kappa A_{x}\right)_{x}, \\
\dot{u} & =F^{*}-u-\frac{1}{L} \int_{0}^{L} A d x
\end{aligned}
$$

where the dimensionless parameters are

$$
L=\frac{\varepsilon l}{d}, \quad \kappa=\frac{D}{d[u]}, \quad F^{*}=\frac{F_{m}}{F_{c}},
$$

and, in keeping with (2.46), we have defined

$$
k=\frac{1}{u_{c}^{2} d} .
$$

The dimensionless drag $F$ is given for laminar flow by $F=u$, and Blasius's law for turbulent flow (2.6) is

$$
F \approx \frac{[R e]^{3 / 4}}{200} u^{7 / 4} \approx 1.66 u^{7 / 4}
$$

for $u \gtrsim 1$, where the Reynolds number scale is taken to be 2300 , by choice of $[u]$. This yields

$$
A \approx 1.66 u^{7 / 4}-u \text { for } u \gtrsim 1
$$

for the upper part of the curve in Figure 2 . Over the range $1 \lesssim u \lesssim 4$, corresponding to Reynolds numbers up to $10^{4},(2.59)$ is well approximated by $A \approx u^{2}-\frac{1}{4} u$, i.e., $u \approx \frac{1}{8}+\left(A+\frac{1}{64}\right)^{1 / 2}$ for $0.75 \lesssim A \lesssim 15$, and this in turn is well approximated by $u \approx 0.7+0.6 A^{0.64}$. Our choice for $\bar{u}(A)$ is thus motivated by this expression, with an extra term $\propto 1 / A^{2}$ added (in order to provide the unstable branch in Figure 2, and to 
ensure linear decay at small $A$ ), with a coefficient $\delta$ chosen so that $\bar{u}$ has a minimum at $\bar{u}=1$. Thus, we choose

$$
\bar{u}(A)=a+b A^{s}+\frac{\delta}{A^{2}},
$$

and the values of the parameters motivated by the above discussion are

$$
a=0.7, \quad b=0.6, \quad s=0.64, \quad \delta=0.0035 .
$$

It is a happy fact that the resulting decay rate of small disturbances $A \ll 1$ is $\dot{A} / A \approx-\delta$, which corresponds to the slow viscous decay rate $(\delta \sim \varepsilon \approx 0.014)$, as is appropriate. Figure 16.3 of Schlichting (1979) indicates that a natural pipe length scale over which the intermittency factor $\gamma$ grows is $l / d \sim 300$. For such pipe lengths (in a $1 \mathrm{~cm}$ diameter pipe this is $l=3 \mathrm{~m}$ ), $L \approx 4.5$, so that values of $L \gtrsim 1$ are appropriate.

The choice of the advection velocity $U$ and the diffusivity $\kappa$ are as follows. For turbulent flow, we expect $\mu_{T} \sim \rho[u] d$; hence (2.43) implies $\kappa \sim 1$, but for small $A$ it should be $O(\varepsilon)$, corresponding to the laminar viscosity term. The simplest choice compatible with these criteria is

$$
\kappa=A+\varepsilon,
$$

although in our numerical illustrations we will be content with the choice $\kappa=1$. For the advection velocity, the choice $U=u$ corresponds to the mean flow. We define

$$
U=u-V(A)
$$

and will find that the corrective term to the mean flow allows us to include a realistic description of puffs within this simple theory, providing $V>0$, as we expect.

\section{Analysis.}

3.1. Constant velocity. We begin our analysis of (2.55) by supposing that the inlet velocity $u$ is constant. We write the amplitude equation in the form

$$
\varepsilon\left(A_{t}+U A_{x}\right)=f(A ; u)+\varepsilon^{2}\left(\kappa A_{x}\right)_{x},
$$

where $U=u-V(A), \kappa=A+\varepsilon$, and

$$
f(A ; u)=(u-a) A^{3}-b A^{3+s}-\delta A .
$$

The form of slugs. Clearly, $A$ evolves locally over a rapid time scale of $O(\varepsilon)$ to a steady state of (3.1), i.e., to $A=0$ (laminar) or to the stable (turbulent) branch of $u=\bar{u}(A)$ (see Figure 2). As shown in Figure 5, $f$ is pseudocubic, and for $u>1$ (the minimum value of $\bar{u}(A)$, corresponding here to $R e=2300$ ) there are three roots of $f$ : $A=0$ and the stable and unstable branches, which are defined to be $A=A_{m}(u)$ and $A_{M}(u)$, respectively.

We change coordinates to the local moving frame coordinates $(X, T)$ given by

$$
x=u t+\varepsilon X, \quad t=\varepsilon T,
$$

whence (3.1) becomes

$$
A_{T}-V(A) A_{X}=f(A)+\left\{\kappa(A) A_{X}\right\}_{X} .
$$




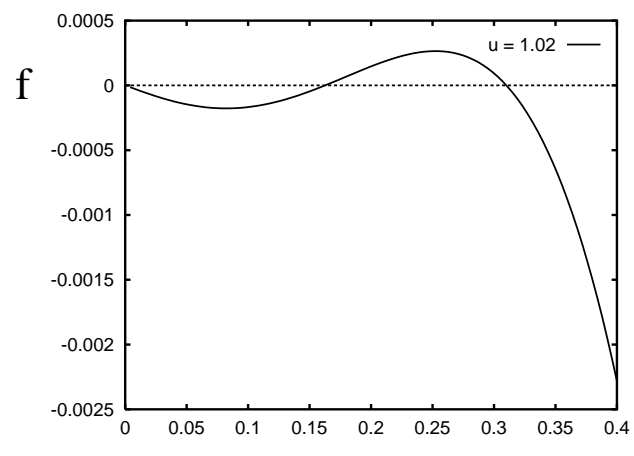

A

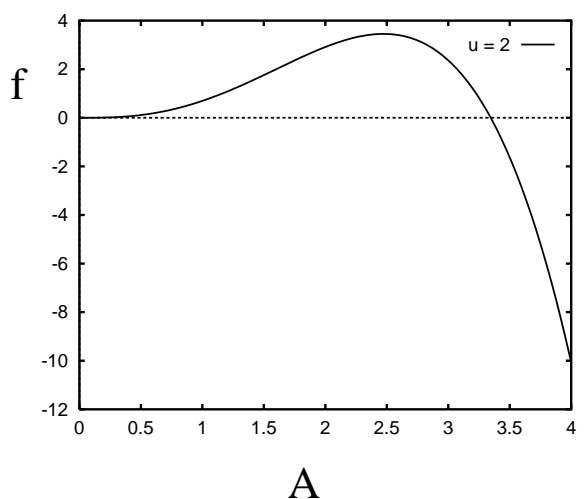

A

FIG. 5. The function $f(A, u)$ for $u=1.02$ and $u=2$. Note the different vertical and horizontal scales.

It is well known from reaction-diffusion theory (Murray 1993) that a local perturbation $A>A_{m}$ will evolve to a slug within which $A=A_{M}$, and the front and rear move outward at speeds $v_{+}$and $v_{-}$, respectively, given by

$$
v_{ \pm}=\frac{\int_{0}^{A_{M}} \kappa(A) f(A) d A \mp \int_{-\infty}^{\infty} \kappa(A) V(A) A^{\prime 2} d z}{\int_{-\infty}^{\infty} \kappa(A) A^{\prime 2} d z}
$$

where $A(z)$ is the wave front solution of

$$
-v_{ \pm} A^{\prime} \mp V(A) A^{\prime}=f(A)+\left\{\kappa(A) A^{\prime}\right\}^{\prime}
$$

together with

$$
\begin{array}{lll}
A \rightarrow A_{M} & \text { as } & z \rightarrow-\infty, \\
A \rightarrow 0 & \text { as } & z \rightarrow+\infty .
\end{array}
$$

Note that the two wave front profiles are different if $V \neq 0$ (and then $v_{+} \neq v_{-}$). The coordinate $z= \pm X-v_{ \pm} T$ is the wave front variable. From (2.30), we define the front and rear slug boundary speeds $u_{f}$ and $u_{r}$ as

$$
\begin{aligned}
& u_{f}=u+v_{+}, \\
& u_{r}=u-v_{-} .
\end{aligned}
$$

If $V=0$, so that the wave profiles are symmetric, then $v_{+}=v_{-}$and the mean slug speed is that of the mean flow; however, this is not observed.

The data in Figure 7 of Wygnanski and Champagne (1973), and in Figure 4.15 of Lindgren (1957), can be used to constrain, to some extent, our choices for $\kappa(A)$ and, particularly, $V(A)$. First, note that if $v_{+}+v_{-}<0$, then slugs contract and are not viable. If $V$ is small, then this occurs if $\int_{0}^{A_{M}} \kappa(A) f(A) d A<0$. We can see from the form of Figure 5 that there will be a critical value of $u=u^{*}>1$ where $\int_{0}^{A_{M}\left(u^{*}\right)} \kappa(A) f\left(A ; u^{*}\right) d A=0$, so that slugs are only viable if $u>u^{*}$. For $V \neq 0$, we can expect $u^{*}$ to depend on $V$. In Wygnanski and Champagne's data, the corresponding Reynolds number is 2700 , whence $u^{*} \approx 1.17$ (based on a Reynolds number scale of 2300). This is also the minimum value for which Lindgren provides identifiable slug data. For $u>u^{*}$, the data indicate that $u_{f} / u$ and $u_{r} / u$ are functions 

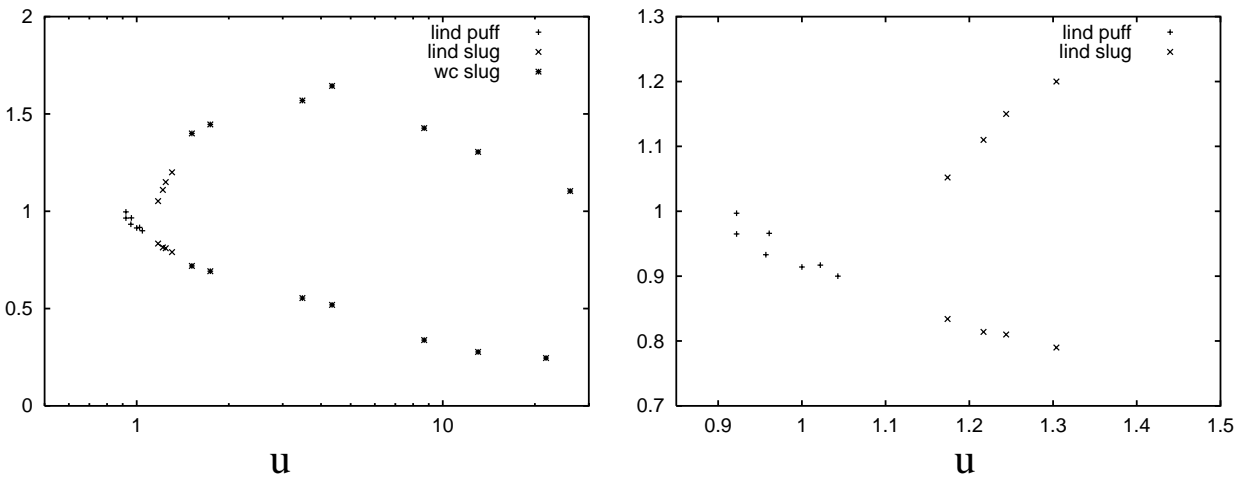

FIG. 6. Estimates for the relative front and rear speeds $u_{f} / u$ and $u_{r} / u$, redrawn from the data of Wygnanski and Champagne (1973) and Lindgren (1957). On the right is a close-up of (some of) Lindgren's data.

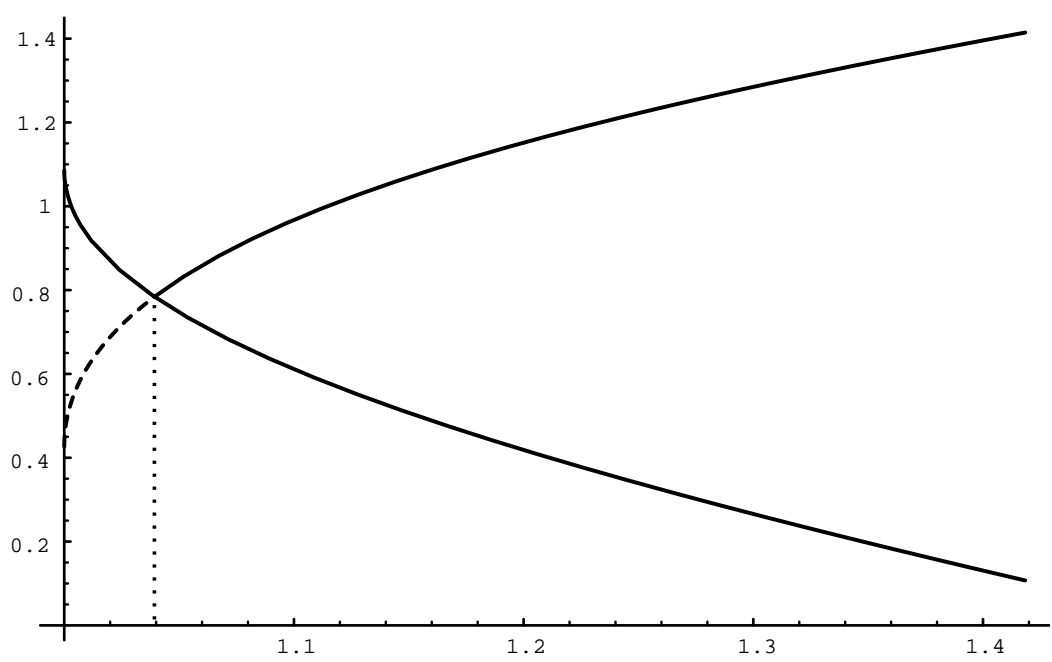

FIG. 7. Front and rear relative wave speeds $u_{f} / u$ and $u_{r} / u$, as a function of $u$. The parameter values used are $b=0.6, s=0.64$, and $\delta=0.1$, with the drift velocity $V(A)$ being taken as $V=0.4 A$ and the diffusivity $\kappa=1$. The critical value at which the two speeds coalesce is approximately 1.04 in this case, and the critical relative speed is about 0.8 . With $[R e]=2300$, this corresponds to a value of $R_{k}$ (in Lindgren's (1957) notation) of 2392.

of $u$, as shown in Figure 6; they appear to coalesce when $R e=R_{k} \approx 2400$, at a value of about 0.9 . We have solved the travelling wave equation numerically, to reproduce data equivalent to that shown in Figure 6. Figure 7 shows the results of these calculations; they are in good qualitative agreement with Lindgren's (1957) results.

A feature of a slug is its sharp interface. In the travelling wave solutions of (3.6), this sharpness is represented both by the short wavelength $\varepsilon$ (corresponding to a tube diameter) and also by the fact that (approximately) $\kappa \rightarrow 0$ as $A \rightarrow 0$; the diffusivity is degenerate and this causes $A$ to reach (approximately) zero in a finite distance.

It is straightforward to compute the value of $u^{*}$ (if $V=0$ ) explicitly from the 
expression (3.2) for $f$. We suppose $\kappa=A$, so that $A_{M}$ and $u^{*}$ are determined from

$$
\begin{aligned}
& (u-a) A^{3}-b A^{3+s}-\delta A=0, \\
& \frac{(u-a)}{5} A^{5}-\frac{b}{5+s} A^{5+s}-\frac{\delta A^{3}}{3}=0
\end{aligned}
$$

from these we find

$$
\begin{aligned}
& A_{M}=\left[\frac{2 \delta(5+s)}{3 s b}\right]^{\frac{1}{2+s}} \\
& u^{*}=a+5(2+s)\left(\frac{\delta}{3 s}\right)^{\frac{s}{2+s}}\left(\frac{b}{2(5+s)}\right)^{\frac{2}{2+s}},
\end{aligned}
$$

and for the numerical values given in $(2.61)$, we find $u^{*} \approx 1.01, A_{M} \approx 0.28$.

In this theory, $u^{*}$ is the critical velocity required for slug propagation. Because $\delta \ll 1$, we see that, for $u>u^{*}$, the unstable zero $A_{m}$ of $f$ is approximately given by

$$
A_{m} \approx\left(\frac{\delta}{u-a}\right)^{1 / 2} .
$$

Note the similarity to the fitted curve in Figure 4. Thus small amplitude disturbances of $O\left(\delta^{1 / 2}\right)$ spontaneously generate slugs, which grow as they propagate.

The form of puffs. When $u<u^{*}$, slugs cannot be maintained. In this case, large disturbances will cause puffs to propagate. These migrate slowly backwards relative to the mean flow, with a sharp trailing edge and a diffuse advancing boundary. In order to explain their characteristics, we observe first that when $u<u^{*}$, then $f$ is small and $A_{M}$ is as well (see Figure 5). In fact, (3.10) suggests that we write

$$
A=\delta^{\frac{1}{2+s}} \alpha, \quad u-a=\delta^{\frac{s}{2+s}} w
$$

in this case, and then

$$
\begin{aligned}
f(A, u) & =\delta^{\frac{3+s}{2+s}} F(\alpha, w), \\
F & =w \alpha^{3}-b \alpha^{3+s}-\alpha,
\end{aligned}
$$

and (3.4) is

$$
\alpha_{T}-V\left[\delta^{\frac{1}{2+s}} \alpha\right] \alpha_{X}=\delta F(\alpha, w)+\left\{\kappa\left[\delta^{\frac{1}{2+s}} \alpha\right] \alpha_{X}\right\}_{X} .
$$

Now let us suppose, for example, that $V(A)=c A$ (as in Figure 7$), \kappa(A) \approx A$. If we define the slow time scale $\tau$ by

$$
T=\frac{\tau}{\delta^{\frac{1}{2+s}}}
$$

then $\alpha$ satisfies the approximate equation

$$
\alpha_{\tau}-c \alpha \alpha_{X}=\left(\alpha \alpha_{X}\right)_{X}+\delta^{\frac{1+s}{2+s}} F(\alpha ; w),
$$

which is, to leading order, a degenerate nonlinear diffusion equation of Burgers type. 


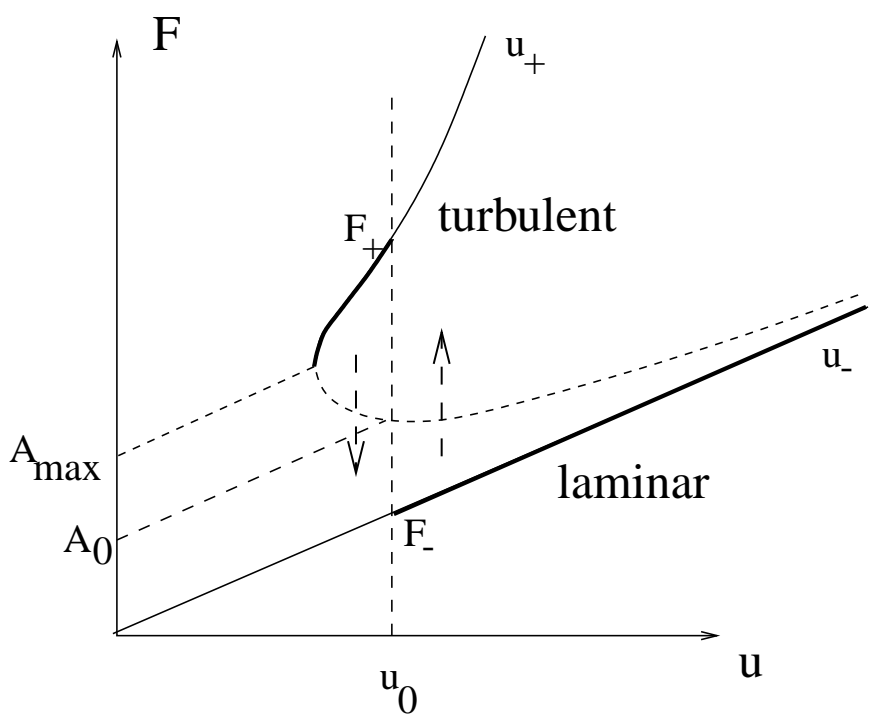

FIG. 8. Diagrammatic representation of intermittency. The inlet disturbance amplitude $A_{0}$ determines a threshold velocity $u_{0}$ on the unstable branch, such that the turbulent branch is stable for $u>u_{0}$ and the laminar branch is stable for $u<u_{0}$, as indicated by the arrows. If the prescribed pressure drop $F^{*}$ lies between the corresponding intersection values $F_{ \pm}$of $u=u_{0}$ with the turbulent and laminar branches, then an intermittent flow will ensue.

Solutions behave as follows. Let $\alpha_{m}=O(1)$ denote the lower positive zero of $F$ in (3.13). For prolonged perturbations to $A$ in excess of $\delta^{\frac{1}{2+s}} \alpha_{m}$, a puff will form. The turbulent flow within the puff will be locally stable, but will eventually disappear by wastage of the profile. However, before this happens, the disturbance amplitude $\alpha$ will evolve over a time scale $\tau \sim 1$, i.e., $T \sim 1 / \delta^{\frac{1}{2+s}}$, according to

$$
\alpha_{\tau}-c \alpha \alpha_{X}=\left(\alpha \alpha_{X}\right)_{X},
$$

and thus into a profile with a sharp upstream (shock) profile and a diffuse downstream profile. The speed of the upstream front, relative to the mean velocity, is negative and of order $d X / d T \sim \delta^{\frac{1}{2+s}}$. All of these features are consistent with puffs. In particular, note that the necessary disturbance amplitude is $O\left(\delta^{\frac{1}{2+s}}\right)$, as opposed to the smaller threshold $O\left(\delta^{1 / 2}\right)$ for slugs. The formation of a backward propagating puff relative to the mean flow relies on the sign of $V$ being positive. If this is the case, then the simple theory based on (3.4) is sufficient to explain many of the pertinent facts concerning slugs and puffs.

3.2. A mechanism for intermittency. The above discussion indicates that the amplitude evolution equation (3.1) can explain both artificially generated slugs and puffs if the flow rate is constant. In order to explain how intermittency can arise spontaneously, we must return to the pressure driven flow model given by (2.55).

Suppose that the inlet disturbance level is $A=A_{0}$. Since $f$ is an increasing function of $u$, there is a unique value $u=u_{0}$ for which $A_{m}\left(u_{0}\right)=A_{0}$. Suppose that $u<u_{0}$. Then $A \rightarrow 0$ in $x>0$ (see Figure 8), and (eventually) $u \rightarrow F^{*}$ from (2.55). However, if $F^{*}>u_{0}$, then at some point $u$ reaches $u_{0}$, and for $u>u_{0}, A \rightarrow A_{M}(u)$ near the inlet: a slug is generated. Again (eventually) $u$ tends to the positive root 




FIG. 9. Mean velocity fluctuations in the solution of (2.55). The parameter values used are $L=4, A_{0}=0.3, \varepsilon=0.05, \kappa(A)=1, U(A, u)=u, a=0.7, b=0.6, s=0.64, \delta=0.1, F^{*}=3$, $u(0)=3.1$. The space step was 0.01 , and the time step was 0.001 .

of $F^{*}=u+A_{M}(u)$. (Note that this is simply the upper branch of the $F(u)$ curve in Figure 1.) But if the corresponding value of $u<u_{0}$, then again $A$ decreases towards zero (a slug is terminated). It is fairly clear that this sequence will continue to oscillate, and also that the dependence of $u$ on the spatial integral of $A$ will cause a finite sequence of slugs to propagate downstream. The intermittency factor $\gamma$ of Rotta (1956) will increase with $x$ due to the spreading of the slugs.

Figure 8 illustrates this description graphically. Observe that the preceding paragraph indicates that $u \rightarrow u_{-}\left(F^{*}\right)$ if $u<u_{0}\left(A_{0}\right)$ and $u \rightarrow u_{+}\left(F^{*}\right)$ if $u>u_{0}\left(A_{0}\right)$, where $u_{ \pm}\left(F^{*}\right)$ are the turbulent and laminar branches of Figure 8. Thus, intermittency should occur, given $F^{*}$ and $A_{0}$, if

$$
u_{+}\left(F^{*}\right)<u_{0}\left(A_{0}\right)<u_{-}\left(F^{*}\right)
$$

in Figure 8. This depends on both the prescribed pressure drop $F^{*}$ and the inlet disturbance amplitude $A_{0}$. The level curves of $A(=F-u)$ are simply lines parallel to the laminar branch $u_{-}$. Furthermore, $u_{0}\left(A_{0}\right)$ is simply the unstable branch of the $\bar{u}(A)$ curve. Thus, given $A_{0}$, we determine $u_{0}$ as the value of $u$ at which the line $F=u+A_{0}$ intersects the unstable branch in Figure 8. So long as $A_{0}<A_{\max }$ (the value of $F-u$ at the nose of the curve), the value $u_{0}$ defines two values $F_{+}$and $F_{-}$on the stable turbulent and laminar branches, respectively. Intermittency then occurs if

$$
F_{-}<F^{*}<F_{+},
$$

which is equivalent to (3.18).

Numerical results. We have solved the system (2.55) numerically, taking $U=u$ and $\kappa=1$. Figure 9 shows the resulting periodic variations in the mean velocity. This 
figure can be favorably compared with Figure 19 of Prandtl and Tietjens (1934). We have not had to tune the model, and so we believe this behavior to be robust. The choice of functions $U$ and $\kappa$ is immaterial to the phenomenon of intermittency; the advection $U$ affects the specific form of puffs, while we avoid the degenerate $\kappa=A$ in order to avoid numerical awkwardness at slug boundaries. Similarly we choose a relatively high value of $\varepsilon(0.05)$ (and hence also $\delta$ ) in order to avoid the demands of excessively small space steps.

Figure 10 shows the space-time evolution of $A(x, t)$ for the same parameter values as used in Figure 9. After an initial transient, a periodic sequence of slugs is generated at some distance from the inlet and propagates downstream towards the outlet.

4. Discussion. Our primary purpose in this paper was to develop a simple model which could predict the intermittent transition to turbulence which is seen in pipe flow, and we have shown that this can be done using the observed fact that there is a sudden increase in friction at the onset of turbulent flow. We associate this with a hysteretic transition between laminar and turbulent states, characterized by a turbulent fluctuation amplitude $A$. We have then used the observed drag law to build the simplest evolution equation for $A$ that is consistent with both the drag law and an inferred linear stability at small amplitudes. We have also shown that this form of amplitude equation is consistent with a one-equation closure of the time-averaged Navier-Stokes equations resembling those of $R_{i j}-\varepsilon$ type (Launder, Reece, and Rodi (1975), Mathieu and Scott (2000)).

The inclusion of realistic diffusive and advective terms then allows us to describe, within the confines of this evolution equation model, phenomena which can be characterized as slugs or puffs, and we have shown that many of their peculiar features arise naturally from the simple ingredients of the model.

In this discussion, we wish to focus further on two particular experimental observations of the parameter ranges in which laminar, intermittent, or turbulent behavior occurs.

Lindgren (1957) identified four particular transition values of the Reynolds number, which he denoted as $\underline{R}, R_{k}, \bar{R}_{k}$, and $\bar{R}$. The value of $\underline{R}$ occurs when the first self-maintaining puffs are seen (i.e., with an identifiable tail velocity). In Lindgren's experiments, this value is about 2050. The value of $R_{k}$ (about 2400) occurs when slugs are first seen (with identifiable fronts), and the value of $\bar{R}_{k}$ (about 2700) is where these become coherent, that is, they do not split as they propagate. Finally, $\bar{R}$ (about 3300?) denotes the onset of fully developed turbulence. The first three of these values correspond in Figure 6 to values $u \approx 0.9, u \approx 1.04$, and $u \approx 1.17$. In our model (cf. Figure 5) we can identify two critical values of $u$ (and hence $R e$ ). Denoting the smaller and larger positive roots of (3.2) by $A_{m}(u)$ and $A_{M}(u)$, respectively, there is a critical value $u_{1}$ at which $A_{m}=A_{M}$, and a larger value $u_{2}$ for which the wave speeds $v_{+}$and $v_{-}$given by (3.6) and (3.7) sum to zero. When $V$ is small, $u_{2} \approx u^{*}$, as discussed following (3.8), and explicit formulae for these critical values are

$$
\begin{gathered}
u_{1}=a+(2+s)\left(\frac{\delta}{s}\right)^{\frac{s}{2+s}}\left(\frac{b}{2}\right)^{\frac{2}{2+s}}, \\
u_{2} \approx u^{*}=a+5(2+s)\left(\frac{\delta}{3 s}\right)^{\frac{s}{2+s}}\left(\frac{b}{2(5+s)}\right)^{\frac{2}{2+s}} .
\end{gathered}
$$

We identify the values of $u_{1}$ with $\underline{R}$, and $u_{2}$ with $R_{k}$. There is no mechanism in our model for slug splitting, and we cannot produce any equivalent for $\bar{R}_{k}$. Finally, the onset of fully developed turbulence at $\bar{R}$ necessarily must depend on the inlet 


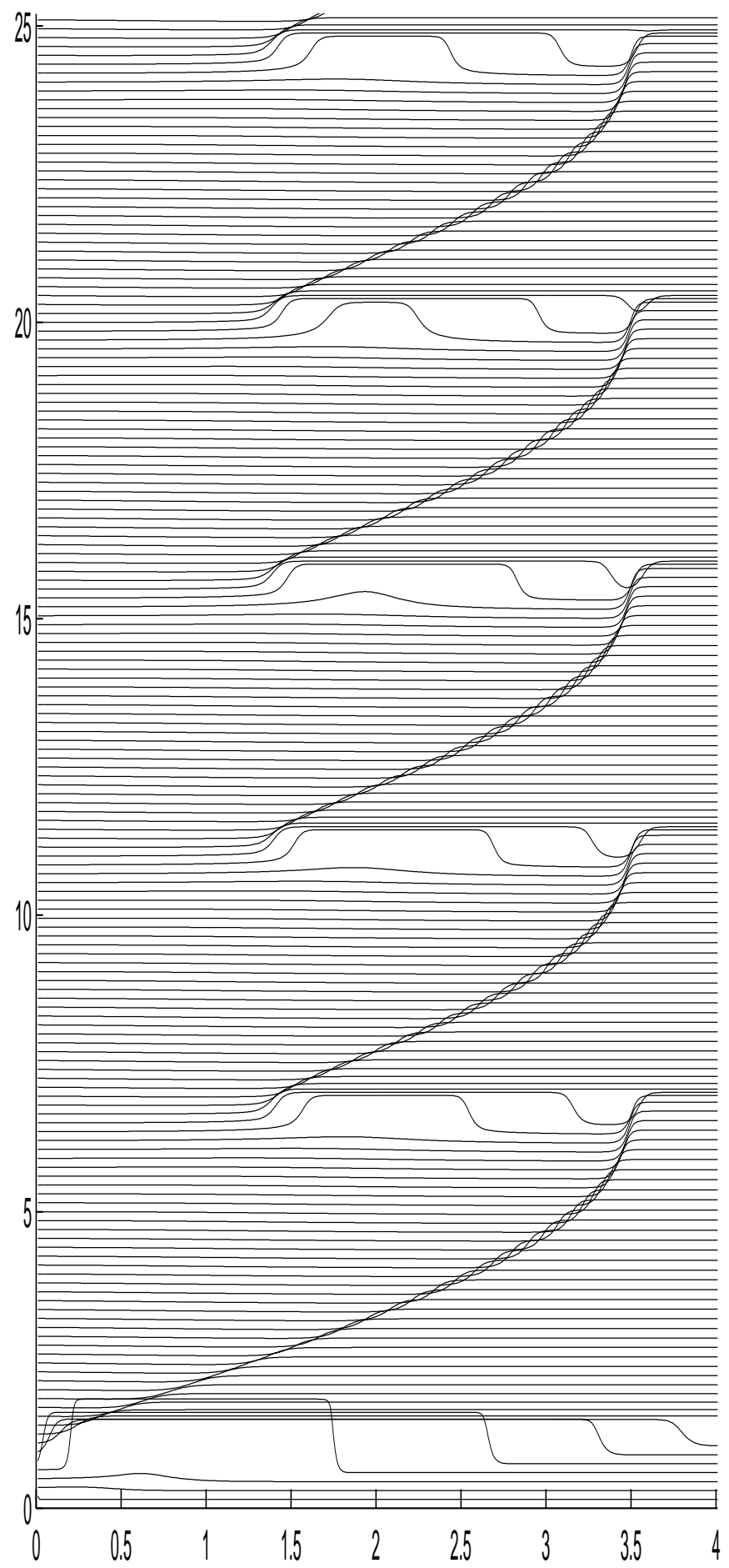

FIG. 10. Space-time plot of $A(x, t)$, using the same parameters as in Figure 9. A sequence of slugs is generated midway down the pipe, each of which grows rapidly and then propagates down the pipe. 
disturbance amplitude level $A_{0}$ (since if this is zero, then the flow may be maintained as laminar indefinitely). In our model, this defines a third transition value $u_{4}$ (the missing $u_{3}$ would correspond to $\left.\bar{R}_{k}\right)$, such that $A_{m}\left(u_{4}\right)=A_{0}$, and thus

$$
u_{4}=a+\frac{\delta}{A_{0}^{2}}+b A_{0}^{s} .
$$

This $u_{4}$ is a monotonically decreasing function of $A_{0}$ in the admissible range $0<$ $A<A_{\max }$, which is where $A_{m}=A_{M}$ and $u_{4}=u_{1}$. Lindgren's apparent value of 3300 corresponds to $u_{4}=1.43$, and when $A_{0}$ is small, (3.11) applies, so that the inferred corresponding inlet disturbance amplitude is $\approx 0.02$. It is also evident that, by adjusting the parameters $b, a, s$, and particularly $\delta$, we could find reasonably accurate values for the predicted $u_{1}$ and $u_{2}$; such an exercise is rather alien to our present purpose, however.

The second comparison we want to make is to the amplitude-velocity transition curve in Figure 2 of Wygnanski and Champagne (1973). This figure plots inlet disturbance amplitude versus Reynolds number and delineates a laminar region from a fully turbulent region. The demarcation boundary essentially consists of two separate curves, between which the flow is intermittent. At high $R e$ (and low $A_{0}$ ), slugs occur in the intermittent region, while at low $R e$ (and high $A_{0}$ ), puffs occur. The slug and puff regions become "uncertain" around $R e=2700$, corresponding in our model to the value $u=u_{2}$.

In our model, the critical inlet amplitude, which separates flows that can be fully laminar from those that can be fully turbulent, is $A_{0}=A_{m}(u)$. However, whether fully developed turbulent or laminar flow can be obtained throughout the pipe depends on the applied pressure drop $F^{*}$, and it is appropriate to use this as the control variable. Denote the turbulent and laminar branches of the pressure drop curve as $F_{T}(u)$ and $F_{L}(u)$, these being given by $(2.60)$ and $F_{L}=u$, respectively. Our predictions for the upper and lower transition curves in $A_{0}-F^{*}$ space are then, for the upper curve,

$$
\begin{aligned}
& F^{*}=F_{T}(u), \\
& A_{0}=A_{m}(u),
\end{aligned}
$$

and for the lower,

$$
\begin{aligned}
& F^{*}=F_{L}(u), \\
& A_{0}=A_{m}(u) .
\end{aligned}
$$

These curves are portrayed in Figure 11, and they show qualitative agreement with Wygnanski and Champagne's (1973) figure (bearing in mind that theirs is a log-linear plot). Indeed we have already seen (compare Figure 4 and (3.11)) that there is some quantitative resemblance also.

The fact that the very simple model used here compares so well with experimental observations is encouraging, but it must be pointed out that the guts of the process are entirely missing, that is to say, the generation of the turbulent chaotic eddies themselves. What we have shown is that some macroscopic features of the transition to turbulence in pipe flow can be understood more or less entirely through the wellfounded postulate of a hysteretic transition between laminar and turbulent "states" of the fluid. Precisely what the turbulent state consists of is not addressed in this theory. 


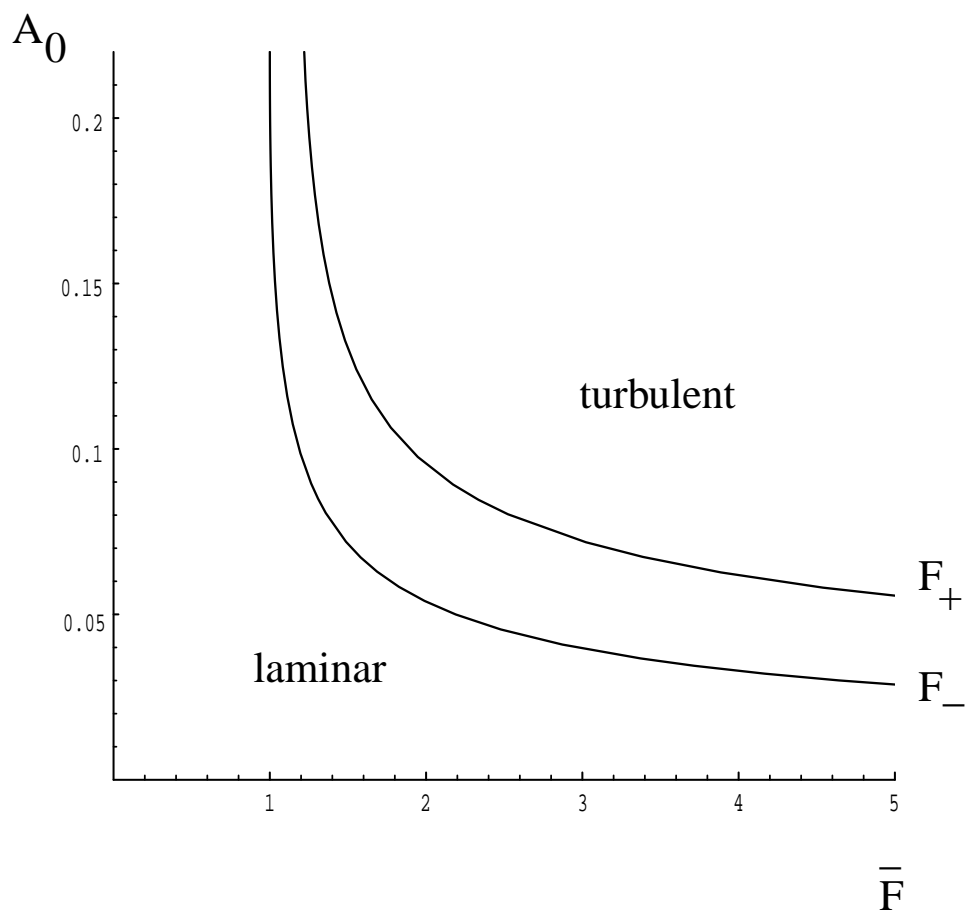

FIG. 11. Parameter space of inlet disturbance $A_{0}$ versus applied pressure drop $F^{*}$, with $b=0.6$, $s=0.64, \delta=0.0035$. Intermittency occurs for points between the two solid curves. Of these, the bottom is $F_{-}=a+b A_{0}^{s}+\delta / A_{0}^{2}$; the top is given by $F_{+}=a+A_{M}+b A_{0}^{s}+\delta / A_{0}^{2}$, where $A_{M}$ is the solution $>A_{0}$ of $b A_{M}^{s}+\delta / A_{M}^{2}=b A_{0}^{s}+\delta / A_{0}^{2}$.

\section{REFERENCES}

H. Blasius (1913), Das Ähnlichkeitsgesetz bei Reibungsvorgängen in Flüssigkeiten, Mitt. Forsch. Arb., 131, pp. 1-39.

T. Bohr And D. A. Rand (1991), A mechanism for localised turbulence, Phys. D, 52, pp. 532543.

J. M. Burgers (1948), A mathematical model illustrating the theory of turbulence, Adv. Appl. Math., 1, pp. 171-199.

A. G. Darbyshire And T. Mullin (1995), Transition to turbulence in constant-mass-flux pipe flow, J. Fluid Mech., 289, pp. 83-114.

A. DAVEY (1978), On Itoh's finite amplitude stability for pipe flow, J. Fluid Mech., 86, pp. 695703.

A. Davey and H. P. F. NGuyen (1971), Finite amplitude stability of pipe flow, J. Fluid Mech., 45 , pp. $701-720$.

R. J. Deissler (1987a), Turbulent bursts, spots and slugs in a generalised Ginzburg-Landau equation, Phys. Lett. A, 120, pp. 334-340.

R. J. DeIsSLer (1987b), Spatially growing waves, intermittency, and convective chaos in an open-flow system, Phys. D, 25, pp. 233-260.

P. G. Drazin and W. H. Reid (1981), Hydrodynamic Stability, Cambridge University Press, Cambridge, England.

S. Goldstein, ED. (1938), Modern Developments in Fluid Dynamics, Vol. 1, Clarendon Press, Oxford, England.

Y.-N. Huang AND Y.-D. Huang (1989), On the transition to turbulence in pipe flow, Phys. D, 37, pp. 153-159.

N. Ітон (1977), Nonlinear stability of parallel flows with subcritical Reynolds numbers. Part 2. Stability of pipe Poiseuille flow to finite axisymmetric disturbance, J. Fluid Mech., 82, pp. 469-479. 
B. E. Launder, G. J. Reece, And W. Rodi (1975), Progress in the development of a Reynoldsstress turbulence closure, J. Fluid Mech., 68, pp. 537-566.

E. R. Lindgren (1957), The transition process and other phenomena in viscous flow, Ark. Fysik, Bd. 12, pp. 1-169.

J. Mathieu And J. Scott (2000), An Introduction to Turbulent Flow, Cambridge University Press, Cambridge, England.

J. D. Murray (1993), Mathematical Biology, 2nd ed., Springer-Verlag, New York.

S. A. Orszag And A. T. Patera (1980), Subcritical transition to turbulence in plane channel flows, Phys. Rev. Lett., 45, pp. 989-993.

S. A. Orszag And A. T. Patera (1983), Secondary instability of wall-bounded shear flows, J. Fluid Mech., 128, pp. 347-385.

L. Prandtl and O. G. Tietjens (1934), Applied Hydro- and Aeromechanics, Dover, New York.

O. REYNOLDS (1883), An experimental investigation of the circumstances which determine whether the motion of water shall be direct or sinuous and of the law of resistance in parallel channels, Proc. Roy. Soc. London A, 35, pp. 84-99.

S. Rosenblat and S. H. Davis (1979), Bifurcation from infinity, SIAM J. Appl. Math., 37, pp. $1-19$.

J. RоттA (1956), Experimenteller Beitrag zur Entstehung turbulenter Strömung im Rohr, Ing.Arch., 24, pp. 258-281.

H. Sakaguchi And H. R. Brand (1996), Stable localized solutions of arbitrary length for the quintic Swift-Hohenberg equation, Phys. D, 97, pp. 274-285.

H. Schlichting (1979), Boundary-Layer Theory, 7th ed., McGraw-Hill, New York.

F. T. Smith And R. J. Bodonyi (1982), Amplitude dependent neutral modes in the HagenPoiseuille flow through a circular pipe, Proc. Roy. Soc. London A, 384, pp. 463-489.

I. J. Wygnanski and F. H. Champagne (1973), On transition in a pipe. Part 1. The origin of puffs and slugs and the flow in a turbulent slug, J. Fluid Mech., 59, pp. 281-335. 